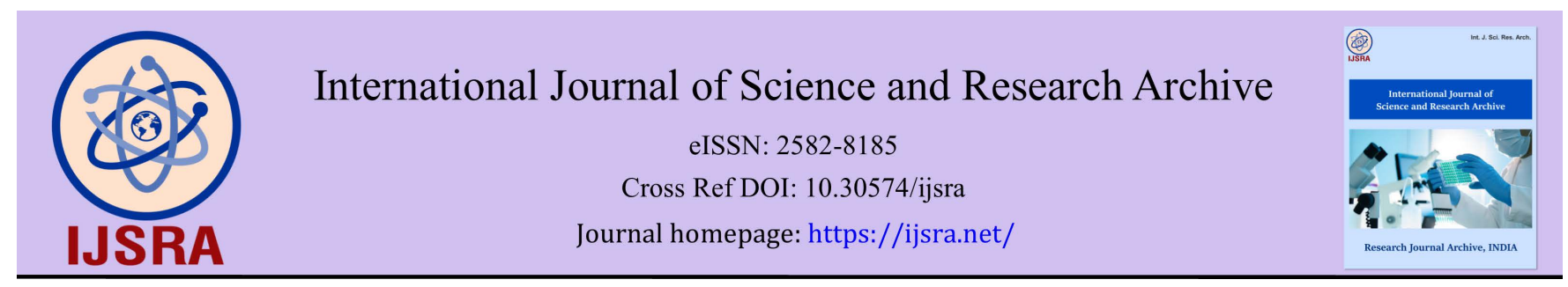

(RESEARCH ARTicle)

\title{
The impact of basic life support training among KIBOGORA district hospital staff: A pilot study
}

Jean C Uwimana*

KIBOGORA district hospital., Anesthesiology, NYAMASHEKE, RWANDA.

International Journal of Science and Research Archive, 2021, 03(02), 218-223

Publication history: Received on 10 September 2021; revised on 21 October 2021; accepted on 23 October 2021

Article DOI: https://doi.org/10.30574/ijsra.2021.3.2.0160

\begin{abstract}
Background: The purpose of BLS is to maintain adequate ventilation and circulation until a means can be used to reverse the underlying cause of cardiac arrest.Worse outcomes are obtained when BLS is being done by non-trained persons.
\end{abstract}

Objectives: To assess the knowledge of BLS among KIBOGORA district hospital staff and the impact of BLS training on retaining BLS skills among clinical and non-clinical KIBOGORA hospital staff.

Materials and Methods: This pilot study was conducted in KIBOGORA hospital. A pre training test was given to every participant and after the training, a posttest was given to all participants. Before completing the pretest questionnaire, each participant had to choose a code made of at least 2 characters and the same code had to appear on the posttest questionnaire of the same participant. A grading system was used to categorize the level of participants' knowledge on BLS skills.

Results: Among 196 trained staff, 40 completed the pre training questionnaire $.82 .50 \%$ of the subjects that completed the pre training questionnaire were clinical staff whereas $17.50 \%$ were non clinical staff. $54.55 \%$ and $33.33 \%$ of the clinical staff had respectively poor and fair knowledge on Basic Life Support (BLS) and only 6.06\% were good at BLS however none of clinical staff was very good or excellent on BLS. All of the non-clinical staff that participated in the study had either a poor or fair knowledge on BLS.

After the training, both clinical and non-clinical staff that completed the pre-training questionnaire, completed the post training questionnaire. $30.30 \%$ of clinical staff that completed the questionnaire were good on BLS.The number of participants with an average or fair knowledge on BLSwere $27.27 \%$ in each category. $6.06 \%$ and $3.03 \%$ were excellent or very good on Basic Life Support. On the other hand, $42.86 \%$ and $28.57 \%$ of non-clinical staff had respectively poor and fair knowledge on BLS.

Conclusion: After the training, knowledge on BLS improved both for clinical and non-clinical staff however more refresher courses are needed to reduce the gap seen in theoretical knowledge as well as practical skills

Keywords: BLS training; KIBOGORA; Pilot Study; Knowledge

\footnotetext{
*Corresponding author: Jean C Uwimana

KIBOGORA district hospital., Anesthesiology, NYAMASHEKE, RWANDA.
} 


\section{Introduction}

Basic life support (BLS) and advanced cardiac life support (ACLS) are part of cardiopulmonary resuscitation (CPR). Invented in 1960, CPR is a simple but effective procedure that allows almost anyone to sustain life in the early critical minutes after cardiac and respiratory arrest [1].

The purpose of BLS is to maintain adequate ventilation and circulation until a means can be used to reverse the underlying arrest [2]. In most of the cases, there is a high probability that a person with cardiac arrest does not receive CPR presumably due to a lack of competence of the bystanders witnessing the emergency [3].And in order to provide high-quality CPR, it is important that responders act as a high-performance team as this forms the cornerstone of any successful resuscitation event [4]. It is expected that health care professionals should have a

sound knowledge about BLS as they encounter an emergency situation very often [5]. The most important items of BLS that are called "the chain of survival" and some other simple first aid techniques that are present in BLS courses and guidelines such as the management of choking and the recovery position $[6,7,8]$ have to be well known and followed when the necessity to apply BLS skills arises. However sometimes that is not the case and worse outcomes are obtained when BLS is being done by non-trained persons.

In previous years, some anesthesia providers at KIBOGORA district hospital trained clinical and non-clinical KIBOGORA district hospital staff on BLS. However, there were no frequent refresher trainings on BLS skills to help KIBOGORA hospital staff to retain BLS skills over time as the ability to perform BLS correctly decreases with the time elapsed since training [3]. In health professionals, it is recommended that there should be no more than 3-6 months between training and refresher sessions and that refresher training should ideally occur monthly.[3]Before We started our training almost 2 years had elapsed since the last session of BLS training or refresher training were done at KIBOGORA hospital.

This study has been conducted to assess the knowledge of BLS among KIBOGORA district hospital staff and the impact of BLS training on retaining BLS skills among clinical and non-clinical KIBOGORA hospital staff.

\section{Methods}

This was a pilot study conducted in December 2019 among KIBOGORA hospital clinical and non-clinical staff from different departments. At the time of this study, all KIBOGORA district hospital staff were 236.Before starting the study, We planned to recruit 45 participant showever 5 participants had incomplete data and they were thus excluded from our study. A pre training test was given to all the participants and after the training, they were given a posttest. Before completing the pretest questionnaire, each participant had to choose a code made of at least 2 characters and the same code had to appear on the posttest questionnaire of the same participant. A grading system was used to categorize the level of participants' knowledge on BLS skills. We used 6 grades. Participants were awarded excellent, very good, good, average, fair and poor when they got $\geq 80 \%, 70-79 \%, 60-69 \%, 50-59 \%, 41-49 \%$, $\leq 40 \%$ respectively. Descriptive statistics were used for analysis and data were presented as distribution or percentages.

\section{Results}

We trained 196 KIBOGORA district hospital staff and 45 among them participated in the study. 5 of the participants had incomplete responses and were excluded from the study. $82.50 \%$ of the subjects that completed the questionnaire were clinical staff whereas $17.50 \%$ were non clinical staff as figure 1 .

$54.55 \%$ and $33.33 \%$ of the clinical staff that participated in the study had respectively poor and fair knowledge on Basic Life Support (BLS) and only $6.06 \%$ were good at BLS however none of clinical staff was very good or excellent on BLS as shown in the figure2.

All of the non-clinical staff that participated in the study had either a poor or fair knowledge on BLS as it is shown below in figure 3. 


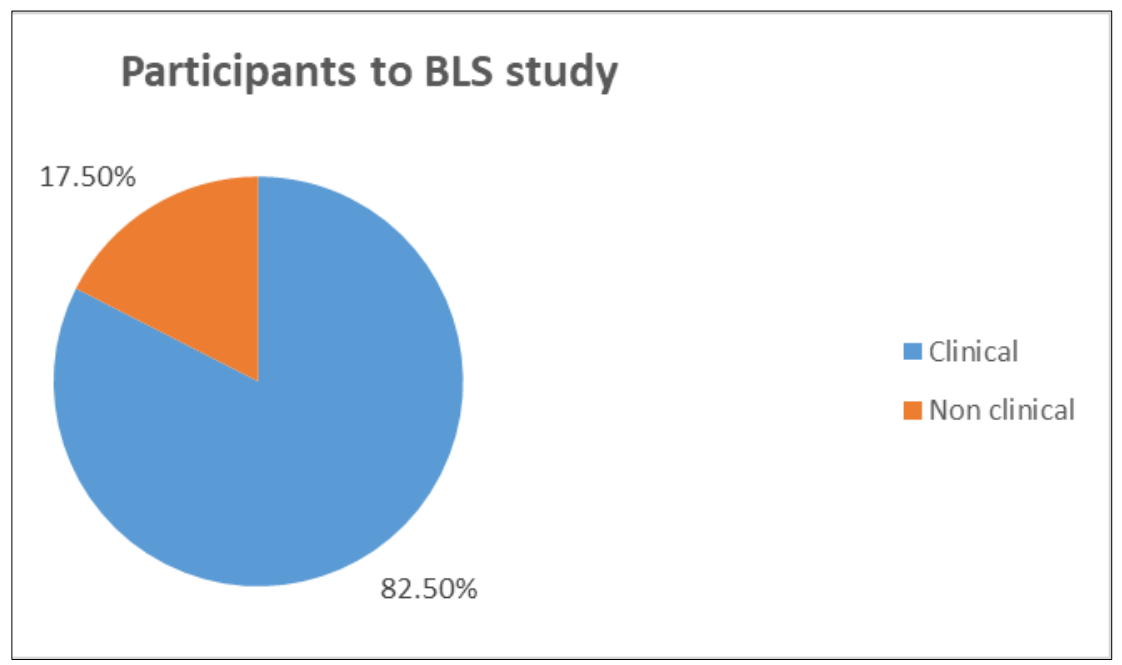

Figure 1 Clinical and non-clinical staff that participate to BLS study

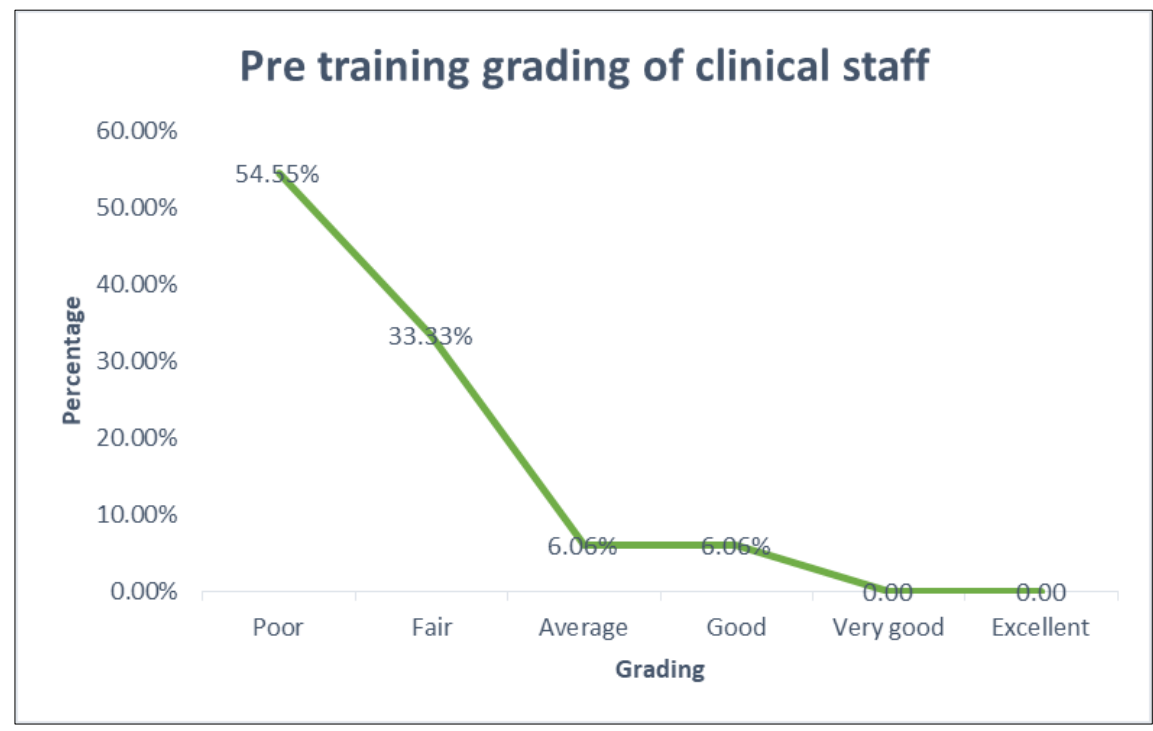

Figure 2 Pre-training grading of clinical staff

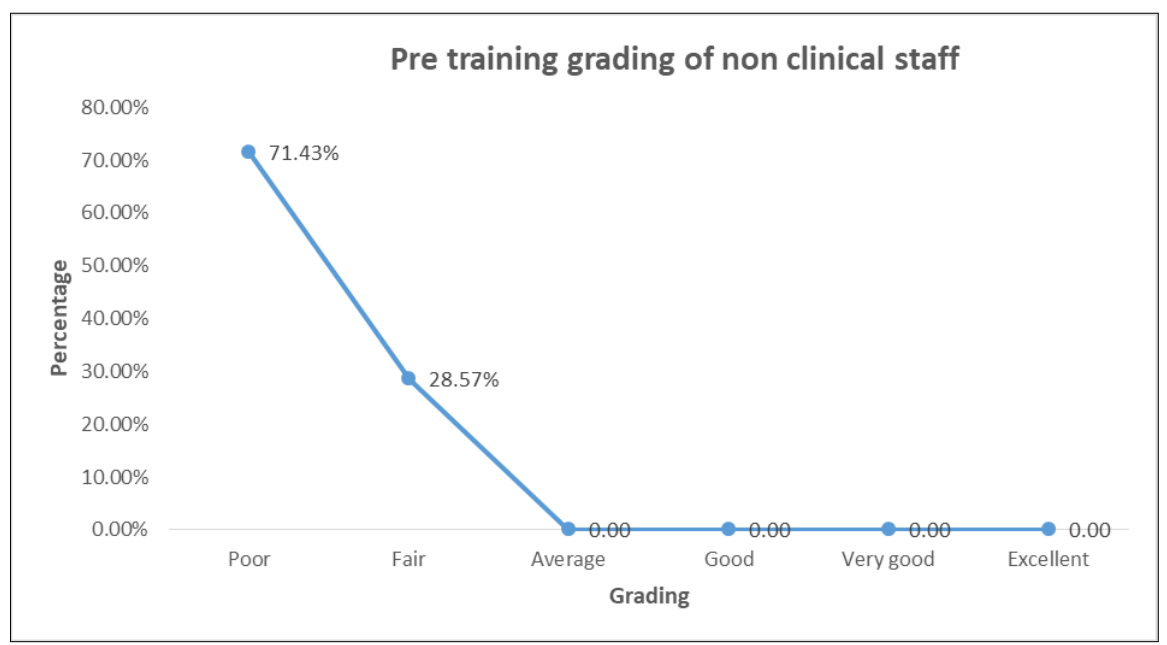

Figure 3 Pre-training grading of non-clinical staff 
After the training, both clinical and non-clinical staff that completed the pre-training questionnaire completed the post training questionnaire.30.30\% of clinical staff that completed the questionnaire were good on BLS. The number of participants with an average or fair knowledge on BLS were $27.27 \%$ in each category. $6.06 \%$ and $3.03 \%$ were excellent or very good on Basic Life Support as shown below in the figure 4.

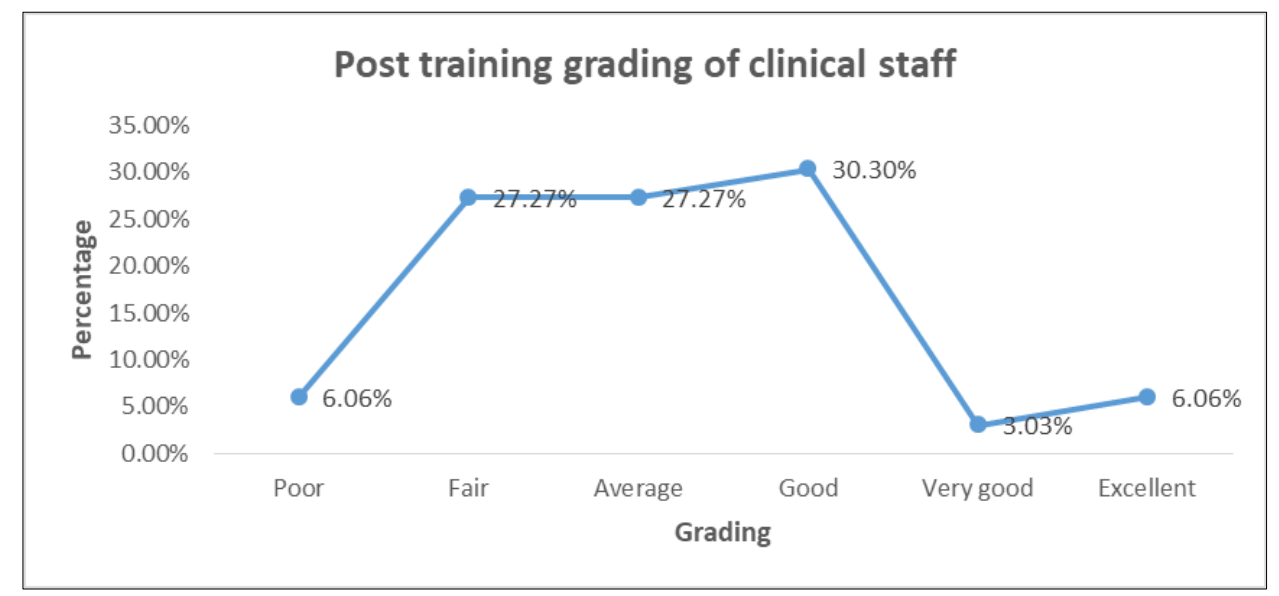

Figure 4 Post training grading of clinical staff

After the training, $42.86 \%$ and $28.57 \%$ of non-clinical staff had respectively poor and fair knowledge on BLS as shown below in figure 5 .

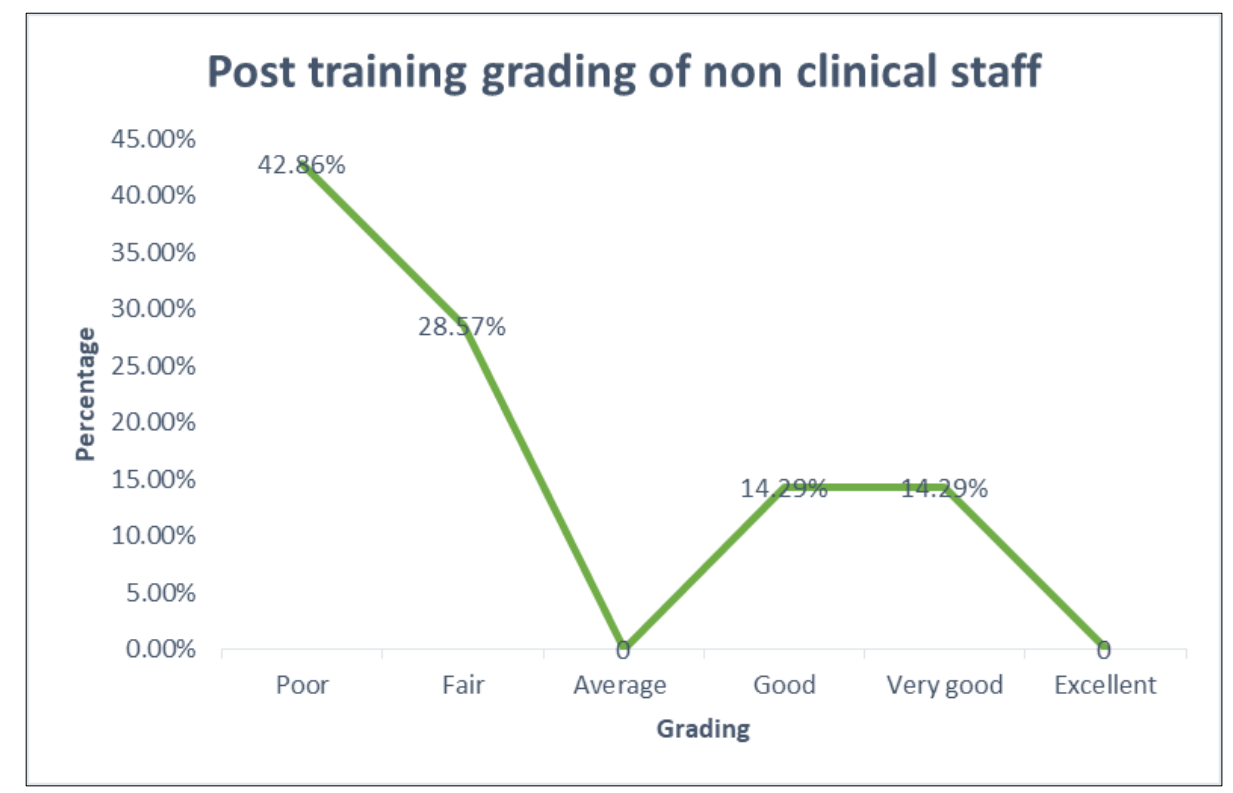

Figure 5 Post training grading of non-clinical staff

\section{Discussion}

This study was done to assess the knowledge of BLS among KIBOGORA district hospital staff and the impact of BLS training on retaining BLS skills among clinical and non-clinical KIBOGORA hospital staff. Before starting the training, $87.88 \%$ of clinical staff and $100 \%$ of non-clinical staff that participate in the training had a poor or fair knowledge on BLS. Compared to a study done in Pakistan in 2018 by Irfan et al. on knowledge of basic life

support in health professionals for which 58.3\% of health professionals had an inadequate knowledge on BLS [9], and another study done by Mendhe HG et al. in 2013 on knowledge, attitude and practice study on cardiopulmonary resuscitation among medical and nursing interns for which $36 \%$ of medical interns had poor knowledge score and $46 \%$ of nursing interns had poor knowledge score [10],the results of our study show that a very high number of health 
professionals had inadequate knowledge and this showed the urgency of the training on BLS at KIBOGORA district hospital.

In their study, they conducted between December 2012 and May 2013,in the emergency services for adults of the hospitals of Murcian Health Service on assessment of the knowledge level and its relevance in terms of CPR in medical personnel, Sanchez Garcia et al. found that $52.5 \%$ of clinical staff passed the BLS test [11] that is they got a score above $50 \%$.Compared to our study for which only 12,1\% of clinical staff passed the BLS test, clinical staff in Murcia had a better knowledge on BLS than KIBOGORA hospital staff. The findings from another study that was done in Portugal by Carla sa Couto and Abel Nicolau[12]revealed better results than the ones from our study and this highlighted the need to train adequately KIBOGORA hospital staff on BLS.

Knowledge of KIBOGORA hospital staff on BLS improved. The studies conducted in other settings like the one done in Malaysia by Wan Jusoh et al. on the effectiveness of modified basic life support module on knowledge, attitude, and performance of cardiopulmonary resuscitation among university students or the other one done in Turkey by Terzi B, et al.on Evaluation of Basic Life Support Training Program Provided for Nurses in A University Hospital showed that the training of BLS was effective as evidenced by the post intervention[13]or the posttest[14]results. Another study done by g. Bielec, p. Klajman, a. Peczak-graczyk on Effectiveness of Basic Life Support Instruction in Physical Education Students showedthat the students improved their knowledge on BLS.[15] However, despite the fact that KIBOGORA staff improved their theoretical knowledge and skills on BLS, 33.33\% of clinical staff and $71.43 \%$ of non-clinical staff still had a poor or fair knowledge on BLS. Therefore, more training sessions or refresher courses are recommended to help KIBOGORA staff to keep improving their theoretical knowledge and technical skills on BLS.

Clinical and non-clinical staff are required to have both theoretical knowledge and practical skills on BLS to improve survival rate for a patient with cardiac arrest. Currently, at KIBOGORA hospital moststaff do not have adequate knowledge on BLS.The hospital resuscitation committee in collaboration with the hospital administration is organizing training sessions or refresher courses every 6 months to get the staff updated on BLS.All KIBOGORA hospital staff should get BLS training as part of their orientation program as they may be the first one(s) to notice cardiac arrests and their timely interventions when cardiac arrests occur will help to improve patients 'survival rate.

Although previous studies have recommended that there should be no more than 3-6 months between training and refresher sessions [3], the gap seen in theoretical knowledge as well as practical skills on BLS can also be reduced through conducting monthly rounds in different departments to assess both clinical and non-clinical staff of KIBOGORA about their confidence level and competence to perform BLS. The information on the level of confidence and competence will help to know how somebody will likely react in case of emergency and it will probably justifies the need to plan for refresher courses.

\section{Conclusion}

Based on the results from this study, there is still much that need to be done to improve both theoretical and practical skills of KIBOGORA staff on BLS. The hospital administration as well as the hospital resuscitation committee can work on how to organize frequent rounds in different departments to assess the level of confidence and competence of different staff as this may help in planning for more refresher courses.

\section{Compliance with ethical standards}

\section{Acknowledgments}

Gilbert Ndayegamiye, Anesthetist, KIBOGORA district hospital for logisticsand valuable scientific inputs during the training.

\section{Disclosure of conflict of interest}

The author declares no competing interests.

\section{Statement of informed consent}

Informed consent was obtained from all individual participants included in the study. 


\section{Funding statement}

Self-sponsorship. Other Support was provided solely from the hospital sources.

\section{References}

[1] Akshatha Rao Aroor, et al. Awareness about basic life support and emergency medical services and its associated factors among students in a tertiary care hospital in South India. Journal of Emergencies, Trauma, and Shock. 2014; I 7: 3.

[2] AJ Handley. Basic Life Support. British Journal of Anesthesia. 1997; 79: 151-158.

[3] Silvia Aranda-Garcia, et al. Basic Life-Support Learning in Undergraduate Students of Sports Sciences: Efficacy of 150 Minutes of Training and Retention after Eight Months. Int. J. Environ. Res. Public Health. 2019; 16: 47-71.

[4] The Canadian Red Cross Society. Basic Life Support Field Guide. 2018.

[5] Shahabe A. Saquib et al. Knowledge and Attitude about Basic Life Support and Emergency Medical Services amongst Healthcare Interns in University Hospitals: A Cross-Sectional Study. Emergency Medicine International. 2019.

[6] Fatima Abbas, et al. Peers versus professional training of basic life support in Syria: arandomized controlled trial. BMC Medical Education. 2018; 18: 142.

[7] National Healthcare Provider Solutions. Basic Life Support: Provider Handbook.1st edition. 2016.

[8] New York State's Bureau of Emergency Medical Services and Traumasystems. Statewide Basic Life Support Adult and Pediatric Treatment Protocols. 2019.

[9] Babar Irfan, et al. Current state of knowledge of basic life support in health professionals of the largest city in Pakistan: a cross-sectional study BMC Health Services Research. 2019; 19: 865.

[10] Mendhe HG, Burra L, Singh D, Narni H. Knowledge, attitude and practice study on cardiopulmonary resuscitation among medical and nursing interns. Int J Community Med Public Health. 2017; 4: 3026-30.

[11] Sanchez Garcia, et al. Assessment of the knowledge level and its relevance in terms of CPR in medical personnel of the hospital emergency medical system of the Autonomous Community of the Region of Murcia. Enfermería Global. 2015.

[12] Carla Sá-Couto, Abel Nicolau. General Public's Knowledge Regarding Basic Life Support: A Pilot Study with a Portuguese Sample.Acta Med Port. 2019; 32(2): 111-118.

[13] Wan Jusoh, et al. The effectiveness of modified basic life support module on knowledge, attitude, and performance of cardiopulmonary resuscitation among university students.Hong Kong Journal of Emergency Medicine. 2019; 26(3): 156-164.

[14] Terzi Banu, et al. Evaluation of Basic Life Support Training Program Provided for Nurses in A University Hospital. Int J Med Res Health Sci. 2017; 6(6): 70-76.

[15] G Bielec, p Klajman, a Peczak-graczy k. Effectiveness of Basic Life Support Instruction in Physical Education Students-A Pilot Study. Teaching and Learning in Medicine. 2014; 26(3): 252-257. 